\title{
Operators of Read's type are not orbit-reflexive
}

\author{
Jean Esterle
}

\begin{abstract}
We show that the operators of Read's type on the Hilbert space constructed by Sophie Grivaux and Maria Roginskaya cannot be orbit-reflexive
\end{abstract}

Mathematics Subject Classification (2000). Primary 47A16; Secondary 47A15.

Keywords. orbit, reflexivity, projection, operators of Read's type.

In a recent preprint [1], Sophie Grivaux and Maria Roginskaya adapted to the separable Hilbert space the celebrated construction by Read [5] of a bounded operator on $l^{1}$ which does not have any proper invariant closed set. They do not reach the same result, but they manage to construct a nonzero operator $T \in \mathcal{L}(H)$ which possesses the two following properties

1. For every $x \in \mathcal{L}(H)$, the closed orbit $\overline{\operatorname{Orb}(x, T)}:={\overline{\left\{T^{n} x\right\}}}_{n \geq 0}$ of $x$ for $T$ is a closed linear subspace of $H$.

2. If $x, y \in H$, then either $\overline{\operatorname{Orb}(x, T)} \subset \overline{\operatorname{Orb}(y, T)}$, or $\overline{\operatorname{Orb}(y, T)} \subset \overline{\operatorname{Orb}(x, T)}$.

Recall that if $X$ is a Banach space, an operator $T \in \mathcal{L}(X)$ is said to be reflexive if every operator $S \in \mathcal{L}(X)$ such that $\overline{\{p(U) x\}}_{p \in \mathbb{C}(\zeta)} \subset \overline{\{p(T) x\}}_{p \in \mathbb{C}(\zeta)}$ for every $x \in X$ (or, equivalently, such that every closed linear subspace of $X$ invariant for $T$ is invariant for $U$ ) belongs to the closure of the set $\{p(T)\}_{p \in \mathbb{C}[\zeta]}$ with respect to the strong operator topology (SOT). Similarly an operator $T \in \mathcal{L}(X)$ is said to be orbit-reflexive [2] if every operator $U \in \mathcal{L}(X)$ such that $\overline{\operatorname{Orb}(x, U)} \subset \overline{\operatorname{Orb}(x, T)}$ for every $x \in X$ (or equivalently, such that every closed subset of $X$ invariant for $T$ is invariant for $U$ ) belongs to the closure of the set $\left\{T^{n}\right\}_{n \geq 0}$ with respect to SOT.

Various conditions which insure that an operator is orbit-reflexive are given in [3], and it is not that easy to exhibit operators which are not orbit-reflexive. Grivaux and Roginskaya indicate how a slight modification of their construction gives an operator which is not orbit reflexive. On the other hand it is well known that an operator for which the set of closed invariant linear subspaces is linearly ordered by inclusion is never reflexive [4].

The aim of this paper is to show that nonzero operators $T \in \mathcal{L}(H)$ satisfying conditions (1) and (2) above are not orbit-reflexive, and so no modification of the 
construction of Grivaux and Roginskaya is needed to obtain a non orbit-reflexive operator on the Hilbert space. These operators are neither reflexive nor orbitreflexive. Examples of reflexive operators on some Banach spaces which are not orbit-reflexive are given by Muller and Vrsovsky in [3], but the existence of a reflexive operator on the Hilbert space which is not orbit-reflexive seems to be an open problem (the Volterra operator on $L^{2}([0,1])$ provides an easy example of a non-reflexive operator which is orbit-reflexive). Other examples of non orbitreflexive operators on the Hilbert space are given in [3].

\section{Operators of Read's type are not orbit-reflexive}

We introduce the following notion.

Definition 1.1. Let $X$ be a Banach space. An operator $T \in \mathcal{L}(X)$ is said to be of Read's type if the two following conditions are satisfied:

1. For every $x \in \mathcal{L}(X)$, the closed orbit $\overline{\operatorname{Orb}(x, T)}$ of $x$ for $T$ is a closed linear subspace of $X$.

2. If $x, y \in X$, then either $\overline{\operatorname{Orb}(x, T)} \subset \overline{\operatorname{Orb}(y, T)}$, or $\overline{\operatorname{Orb}(y, T)} \subset \overline{\operatorname{Orb}(x, T)}$.

Proposition 1.2. Let $X$ be a Banach space, and let $T \in \mathcal{L}(X)$ be an operator of Read's type.

Assume that $P \in \mathcal{L}(X)$ is a bounded linear projection from $X$ onto $\overline{\operatorname{Orb}(a, T)}$ for some $a \in X$. Then $\overline{\operatorname{Orb}(x, P)} \subset \overline{\operatorname{Orb}(x, T)}$ for every $x \in X$. If, further, $\{0\} \neq$ $\overline{O r b(a, T)} \neq X$, then $P T \neq T P$.

Proof. Since $P^{2}=P$, we have $\overline{\operatorname{Orb}(x, P)}=\{x, P x\}$ for every $x \in X$. If $x \in$ $\overline{\operatorname{Orb}(a, T)}$, then $P x=x$, and $\overline{\operatorname{Orb}(x, P)}=\{x\} \subset \overline{\operatorname{Orb}(x, T)}$. If $x \notin \overline{\operatorname{Orb}(a, T)}$, then $P x \in \overline{O r b(a, T)} \subset \overline{\operatorname{Orb}(x, T)}$. Hence $\overline{\operatorname{Orb}(x, P)} \subset \overline{\operatorname{Orb}(x, T)}$ for every $x \in X$.

Now set $F=\operatorname{Ker}(P)$, and assume that $\{0\} \neq \overline{O r b(a, T)} \neq X$. Let $b \in F \backslash\{0\}$. We have $P b=0$, hence $T^{n} P b=0$ for every $n \geq 0$. On the other hand $b \notin$ $\overline{\operatorname{Orb}(a, T)}$ and so $a \in \overline{\operatorname{Orb}(b, T)}$. This means that there exists a sequence $\left(n_{p}\right)_{p \geq 1}$ of positive integers such that $a=\lim _{p \rightarrow+\infty} T^{n_{p}} b$. Hence $a=P a=\lim _{p \rightarrow+\infty} P T^{n_{p}} b$, and $P T^{n_{p}} b \neq 0=T^{n_{p}} b$ when $p$ is sufficiently large, which shows that $T P \neq$ $P T$.

Corollary 1.3. Let $H$ be the separable Hilbert space, and let $T \in \mathcal{L}(H)$ be an operator of Read's type. Then $T$ is not orbit-reflexive.

Proof. It follows immediately from the definition of SOT that the closure in $\mathcal{L}(X)$ of the set $\left\{T^{n}\right\}_{n \geq 0}$ is contained in the commutant of $T$ for every $T \in \mathcal{L}(X)$ if $X$ is a Banach space. So the corollary follows immediately from the proposition, unless $\overline{O r b(x, T)}=H$ for every nonzero $x \in H$. But such an operator, which would provide a counterexample to the invariant subspace problem, would be trivially not orbit-reflexive. 


\section{References}

[1] S. Grivaux and M. Roginskaya, On Read's type operators on Hilbert spaces, Int. Math. Res. Notices 2008, 2008:rnn083-42.

[2] D. Hadwin, E. Nordgren, H. Radjavi, P. Rosenthal, Orbit-reflexive operators, J. London Math. Soc 34 (1986), 111-119.

[3] V. Muller and L. Vrsovsky, On orbit reflexive operators, J. London Math. Soc., to appear.

[4] H. Radjavi and P. Rosenthal, Invariant subspaces, Springer-Verlag, Berlin, Heidelberg, New-York, 1973.

[5] C.J. Read, The invariant subspace problem for a class of Banach spaces II. Hypercyclic operators, Israel J. Math. 63 (1988),1-40.

Jean Esterle

Université de Bordeaux

IMB, UMR 5252

351, cours de la Libération

33405-Talence-Cedex

France

e-mail: esterle@math.u-bordeaux1.fr 